\title{
La kinésiophobie : de l'irrationnel au sensé
}

\section{Kinesiophobia: From Irrational to Meaningful}

\author{
J. Grisart \\ (C) Lavoisier SAS 2020
}

Résumé La kinésiophobie semble devenue une manière habituelle de nommer la peur de la douleur. Cet usage n'est-il pas influencé par ce qui opérationnalise la kinésiophobie, à savoir l'échelle de Tampa ? Cela questionne sa validité eu égard à sa définition. Une exploration qualitative suggère que le sens donné par les patients à la peur mesurée par ce questionnaire n'est pas exclusivement lié au thème de la lésion provoquée par le mouvement. L'échelle de kinésiophobie ne devrait-elle pas être adaptée pour se rapprocher plus de la définition de la kinésiophobie?

Mots clés Peur · Kinésiophobie · Échelle de Tampa Approche qualitative

\begin{abstract}
Kinesiophobia seems to be a common way of naming fear of pain. Is this use not influenced by the contents of the Tampa scale? This questions its validity in terms of its definition. A qualitative exploration suggests that the patient's sense of fear measured by this questionnaire is not exclusively related to the issue of the injury caused by movement. Should not the Tampa scale be adapted to get closer to the definition of kinesiophobia?
\end{abstract}

Keywords Fear · Kinesiophobia · Tampa scale · Qualitative approach

\section{Introduction}

S'il n'est pas toujours facile de savoir pourquoi certaines lombalgies apparaissent, il l'est encore moins de définir pourquoi elles persistent, car l'éventuelle mise en évidence de pathologie ne suffit pas à expliquer la situation. Face à ces difficultés, chacun développe implicitement ou explicitement des explications personnelles pour tenter de compren-

\footnotetext{
J. Grisart ( $\square)$

Cliniques universitaires Saint-Luc, consultation de la douleur chronique, 10, avenue Hippocrate, B-1200 Bruxelles, Belgique e-mail : jacques.grisart@uclouvain.be
}

dre et donc de maîtriser sa douleur et ses répercussions. Les croyances les plus fréquentes sont celles de peur liée à la douleur. Peur et douleur font bon ménage au grand dam du patient. La relation peur-douleur a fait l'objet de nombreux travaux et a été formalisée dans le modèle peur-évitement [1] qui a connu plusieurs remaniements [2-4]. Les éléments cognitifs et motivationnels y ont un rôle crucial, orientant les comportements du patient soit d'oser rester actif malgré la présence de la douleur ou d'éviter ce qui pourrait renforcer la douleur. Diverses peurs paraissent en lien avec la douleur et ont fait chacune l'objet d'un questionnaire. Pour n'en citer que quelques-unes, la crainte que la douleur soit causée et accentuée par le travail et l'activité physique (FABQ) [5], la peur d'éprouver de la douleur (FPQ) [6], la peur d'éprouver de l'anxiété et de la douleur (PASS) [7] et la kinésiophobie (TSK) [8]. Dans le quotidien des activités cliniques, la kinésiophobie est très souvent évoquée. Alors que la kinésiophobie désigne une peur spécifique, elle paraît être devenue dans l'usage courant la manière habituelle de nommer la peur de la douleur, quelle qu'elle soit. Cet article explore si cet usage n'est pas influencé par ce qui opérationnalise la kinésiophobie, à savoir le contenu de l'échelle de mesure. Ce qui en questionne sa validité eu égard à sa définition.

\section{La kinésiophobie, quelques rappels}

Kori et al. [8] ont défini la kinésiophobie comme une peur irrationnelle, excessive et invalidante du mouvement et des activités, résultant du sentiment d'un risque d'une lésion douloureuse. Il s'agit de la peur d'une (nouvelle) lésion suite à un mouvement avec comme répercussion la crainte d'une accentuation de la douleur.

Phobie et douleur ayant des caractéristiques communes (réactivité physiologique accrue, rumination mentale et conduite d'évitement), le modèle thérapeutique recommandé implique une exposition progressive à « l'objet phobique », en l'occurrence des mouvements. Une série de photos (PHODA : Photograph Series of Daily Activities) [9] montrant des mouvements dans diverses activités de la vie quotidienne a été réalisée. Préalablement à l'exposition, les 
patients sont invités à regarder ces photos et à évaluer sur une échelle de 0 à 100 la mesure dans laquelle ils ont l'impression que ce mouvement est néfaste pour leur dos.

La kinésiophobie s'intègre dans le modèle peur-évitement supporté par de nombreuses recherches. Cependant, des essais randomisés [10] rapportent des effets modestes de réduction de la kinésiophobie et du handicap, une faible stabilité des résultats dans le temps et un taux élevé d'abandon. $\mathrm{Ce}$ constat amène des questions. La kinésiophobie, joueraitelle le rôle prépondérant qu'on lui attribue dans la chronicisation de certaines lombalgies?

Au vu de cela et de notre impression d'une surutilisation du terme kinésiophobie, intéressons-nous à la validité de l'outil de mesure de la kinésiophobie. La kinésiophobie est mesurée par l'échelle de Tampa (Tampa Scale for Kinesiophobia-TSK) comportant 17 items. Les items sont évalués sur une échelle de Likert à quatre points allant d'un fort désaccord $($ score $=1$ ) à fortement d'accord $($ score $=4)$. Les scores sur les items 4, 8, 12, 16 sont inversés. L'étendue du score total peut aller de 17 à 69 . Plus le score est élevé, plus la kinésiophobie est forte. Un score supérieur ou égal à 40/60 est considéré comme un score significatif de kinésiophobie. L'échelle de kinésiophobie a un haut degré de validité (notamment Alpha de Cronbach $=0,76$ ). Elle est bien corrélée avec d'autres mesures de peur de la douleur, des mesures d'anxiété et de dépression, ainsi que de dramatisation. Cette échelle est d'emblée apparue comme ayant un bon potentiel pour identifier les patients lombalgiques chroniques dont le handicap est principalement déterminé par la peur spécifique du mouvement qui pourrait blesser et non par l'intensité de la douleur, ou une pathologie organique sous-jacente [11].

Il existe une version réduite [12] dans laquelle les six items les plus faibles sur le plan psychométrique furent retirés. Les propriétés psychométriques de cette version comportant 11 items sont similaires à celles de la version complète.

L'échelle de Tampa est présentée comme unidimensionnelle. En est-il vraiment ainsi ? Des analyses factorielles $[13,14]$ laissent émerger des solutions à deux facteurs : focalisation somatique (FS) et évitement des activités (EA). Ces deux mêmes facteurs sont retrouvés dans la version courte [15]. L'examen de quelques études récentes $[16,17]$ laisse croire que cette solution à deux facteurs n'a pas été adoptée. Le score de kinésiophobie traduit-il uniquement la peur de mouvements qui blessent ou charrie-t-il d'autres éléments ? Ces questions touchent à la définition de la kinésiophobie, à l'usage de ce terme et à la validité de son outil de mesure. Des travaux dans un cadre de recherches qualitatives ont approfondi ces questions. En effet si les études quantitatives reposent sur un grand nombre d'observations, proposant des repères pour la compréhension des phénomènes observés, elles n'éclairent pas suffisamment ces phénomènes au niveau individuel. Or, dans le travail clinique caractérisé par la rencontre avec un individu donné, il est utile de se mettre à l'écoute de sa singularité. L'approche qualitative [18] offre un cadre plus fin visant l'explicitation de la réalité de la personne par l'analyse du discours de cette personne elle-même. Cette perspective amène à interroger la façon dont les individus interprètent des informations et donnent sens à leurs paroles et à leurs actes, ainsi qu'à d'autres aspects du monde avec lesquels ils sont en relation.

\section{Échelle de Tampa et paroles de patients}

À partir d'entretiens semi-directifs auprès de 36 personnes, Bunzli et al. [19] ont exploré les craintes des personnes avec un score élevé à l'échelle de kinésiophobie. Deux croyances prédominantes ressortent :

- la première selon laquelle un mouvement douloureux va blesser le dos (par exemple : «la douleur est vue comme une menace pour la structure de la moelle épinière»; « la peur de provoquer plus de dégâts ou de surcharger un dos déjà fragile »; "l'évitement est un moyen de limiter les blessures »);

- la seconde selon laquelle effectuer un mouvement douloureux va augmenter la souffrance et le déficit fonctionnel.

Les auteurs concluent que l'échelle de kinésiophobie pourrait être décrite plutôt comme une mesure des croyances que les activités douloureuses résulteront en dommages corporels et/ou en une augmentation de la souffrance et/ou de perte fonctionnelle.

Un nouvel entretien d'approfondissement [20] classe les patients en trois groupes. Le récit d'un premier groupe de 14 patients comportait de nombreuses évocations de dommages corporels, de blessures et du caractère néfaste de l'activité physique. Un deuxième groupe de 17 personnes parlait plutôt en termes de perte fonctionnelle. Enfin, un troisième groupe de cinq personnes présentait un profil mixte, évoquant à la fois le risque lésionnel et fonctionnel. Toutefois, l'aspect fonctionnel s'est avéré dominant auprès de deux de ces cinq personnes qui ont rejoint le deuxième groupe (passant de 17 à 19 personnes).

Ces résultats relancent la question concernant le rôle de l'échelle de kinésiophobie ? Les observations recueillies lors de cet entretien ont été mises en lien avec les réponses aux deux dimensions présumées (FS et EA) de l'échelle de kinésiophobie. L'hypothèse est faite que ceux qui ont été catégorisés dans le groupe « dommage corporel » auraient un score élevé sur la dimension FS, et c'est bien le cas. L'hypothèse est faite que ceux appartenant au groupe «perte fonctionnelle » auraient des scores plus élevés sur la dimension EA. Dans ce groupe, en revanche, la dimension EA n'obtient pas des scores plus élevés que la dimension FS. De manière 
inattendue, les deux groupes (« dommage corporel » et «perte fonctionnelle ») sont égaux en ce qui concerne la dimension «EA ».

Ces observations ravivent le débat concernant la validité de l'échelle de kinésiophobie ainsi que la question des deux facteurs. Or, il s'avère que les deux groupes (« dommage corporel » et «perte fonctionnelle ») sélectionnent les quatre items de l'échelle EA contenant le terme injury. Mais peutêtre les deux groupes, interprètent-ils de façon différente le terme injury ? En effet, il apparaît que les personnes du groupe « dommage corporel » l'interprètent comme signifiant " danger » et les personnes du groupe EA comme « va augmenter la douleur ». Ces résultats suggèrent donc l'existence de deux dimensions au questionnaire et contestent la validité du questionnaire de kinésiophobie, dont la définition met l'accent sur la peur d'être blessé.

Ces travaux illustrent la complémentarité des approches qualitatives cernant les différents motifs de menace et d'évitement [21]. La difficulté de comprendre la douleur, de faire face au caractère imprévisible, incontrôlable et/ou intense de la douleur donne aussi à la douleur une valeur de menace. La peur est également alimentée par les expériences passées de douleurs au dos, par les mauvaises expériences avec des soignants, les messages reçus des professionnels, l'absence de diagnostic ou un manque de clarté dans le diagnostic, par des considérations sociétales à l'égard de la douleur, par les échecs thérapeutiques répétés, par le sentiment d'impuissance et par la crainte de ne pas arriver à certains objectifs dans la vie. La menace que représente la douleur n'est donc pas uniquement liée au risque de lésion. Elle est motivée par différents éléments qui tournent autour de la difficulté d'avoir prise sur l'expérience de la douleur. Le suivi des 36 patients a en effet montré que ceux qui étaient améliorés sur le plan de la peur étaient ceux qui rapportaient un meilleur contrôle sur les aspects somatiques, une attitude d'acceptation, une compréhension de leur douleur, tout cela renforçant leur « pouvoir d'agir ». Ceux qui n'étaient pas améliorés faisaient état d'un faible sentiment de contrôle.

Ce serait le manque de prise sur l'expérience de la douleur (au sens large et non uniquement sur la sensation de douleur) qui détermine un dénominateur commun à ce qui fait menace. La crainte d'une lésion peut y contribuer à côté d'autres raisons. Les travaux de Bunzli et al. [19,20] rappellent qu'une diversité de croyances sous-tend la peur de la douleur et que c'est par leur récit que les patients nous apprennent les raisons pour lesquelles, à leurs yeux, une menace pèse sur eux, fragilisant une gestion « efficace » de leur douleur.

\section{Conclusion}

Cet article propose une réflexion sur la validité du questionnaire de Tampa à partir de l'usage fréquent du terme « kiné- siophobie ». Notre raisonnement a souligné la singularité à saisir le sens de ce qui fait menace aux yeux du patient, en quoi cette saisie est au cœur des réponses du patient à sa situation et soutient le processus thérapeutique. La définition de la kinésiophobie attribuant à la peur un caractère « irrationnel et excessif » ne banaliserait-elle pas la nécessité de découvrir des motifs personnels sous-jacents à la peur ?

Après les considérations exposées ci-dessus, il nous paraît maintenant assez compréhensible que le terme kinésiophobie fasse partie du langage courant des soignants pour parler de peur liée à la douleur. En effet, nos observations personnelles nous permettent de rapporter un usage du mot kinésiophobie dépassant sa définition [8], soit la peur que le mouvement entraine des dégâts corporels accentuant la douleur. Une exploration qualitative de l'échelle de Tampa suggère que la peur mesurée par ce questionnaire est loin d'être exclusivement liée au thème de la lésion provoquée par le mouvement. En outre, le fait que le patient coche les items comportant le terme injury ne veut pas dire qu'il parle nécessairement de «blessure ». Le mot fait l'objet d'interprétations diverses qu'il convient de cerner. L'analyse qualitative rejoignant au plus près la vision de l'individu souligne ainsi la nécessité d'avoir accès au sens donné par la personne. Des travaux questionnant l'unidimensionnalité de l'échelle concluaient à l'existence de deux dimensions ; ainsi, le questionnaire embrasserait plus que la kinésiophobie au sens de sa définition. L'inclusion du terme « irrationnelle » dans la définition de la kinésiophobie aurait-il éloigné de la démarche basique qui consiste à découvrir ce qui existe dans toute conduite, à savoir une bonne part de raisons personnelles inconscientes, mais aussi conscientes ? C'est en cela que le tabula rasa phénoménologique de l'approche qualitative est d'un apport précieux ; recommencer comme si on ne savait pas, pour mieux entendre ce qui se passe dans l'ici et maintenant d'un individu donné et ne pas trop aisément attribuer les répercussions fonctionnelles à de l'évitement induit par la peur de la douleur [22]. C'est essentiel, car le travail clinique s'organise à partir d'une rencontre avec un individu et de sa vision à lui, réclamant une approche centrée sur la personne du patient [23] et non — du moins dans un premier temps sur nos représentations de professionnels de la santé.

Nos propos jettent peut-être un trouble. La crainte de la lésion entraînée par le mouvement ne pèserait donc pas autant sur la réactivation physique des lombalgiques chroniques. Alors quid de l'adéquation d'une remise en mouvement par le protocole d'exposition graduée lié à un score élevé sur l'échelle de Tampa ? Ce protocole n'a-t-il pas été surutilisé du fait du trop grand nombre de faux-positifs ? Quels peuvent être les effets d'un tel protocole, destiné à une problématique phobique, chez des personnes dont les conduites d'évitement sont loin d'être motivées par une phobie liée à la douleur? Les résultats mitigés des travaux sur le traitement de la kinésiophobie ne reflètent-ils pas une 
bidimensionnalité non reconnue de l'échelle ? La morale de l'histoire n'est-elle pas la suivante : tout en ayant des repères théoriques en tête, il convient de prendre le temps d'explorer les représentations singulières de la personne et de construire avec elle une démarche de remobilisation s'appuyant sur ses raisons de bouger. Le sens tapi au sein des gestes, des mouvements et de la posture est l'énergie qui fait (fera) que la motricité soit (ou devienne) fluide ou figée.

Face à l'importante littérature de ces 20 dernières années sur la kinésiophobie [10], on pourrait dire que les quelques travaux de Bunzli et al. [19-21] ne font pas le poids. Cet article n'a d'autre ambition que celle d'ouvrir une réflexion, car nos pratiques doivent s'alimenter à la fois de tout ce qui les conforte et ce qui les contredit pour donner, face à l'âpreté de l'algologie, plus de sens à la construction du fragile fil thérapeutique traversant nos subjectivités et porté par elles. Il est peut-être encore trop tôt pour recommander un reformatage de l'échelle de kinésiophobie. Quoi que... ? Il est permis de commencer à y penser.

Liens d'intérêts : l'auteur déclare ne pas avoir de liens d'intérêts.

\section{Références}

1. Vlaeyen JWS, Linton SJ (2000) Fear-avoidance and its consequences in chronic musculoskeletal pain: a state of the part. Pain 85:317-32

2. Norton PJ, Asmundson GJG (2003) Amending the fearavoidance model of chronic pain: what is the role of physiological arousal? Behav Ther 34:17-30

3. Crombez G, Eccleston C, Van Damme S, et al (2012) Fearavoidance model of chronic pain. The next generation. Clin J Pain 28:475-83

4. Vlaeyen JWS, Crombez G, Linton SJ (2016) The fear-avoidance model of pain. Pain 157:1588-9

5. Waddell G, Newton M, Henderson I, et al (1993) Fear-avoidance beliefs questionnaire (FABQ) and the role of fear-avoidance beliefs in chronic low back pain and disability. Pain 52:157-68

6. McNeil DW, Rainwater AJ 3rd (1998) Development of the Fear of Pain Questionnaire-III. J Behav Med 21:389-410

7. McCracken LM, Zayfert C, Gross RT (1992) Pain anxiety symptoms scale: development and validation of a scale to measure fear of pain. Pain 50:67-73
8. Kori S, Miller R, Todd D (1990) Kinesiophobia: a new view of chronic pain behaviour. Pain Manag 3:35-43

9. Kugler K, Wijn J, Geilen M, et al (1999) The photograph series of daily activities (PHODA). CD-Rom version 1.0. Institute for rehabilitation research and school for physiotherapy. Heerlen, The Netherlands

10. Luque-Suarez A, Martinez-Calderon J, Falla D (2019) Role of kinesiophobia on pain, disability and quality of life in people suffering from chronic musculoskeletal pain: a systematic review. $\mathrm{Br}$ J Sports Med 53:554-9

11. Vlaeyen JWS, Kole-Snijders AMJ, Boeren RGB, van Eek H (1995) Fear of movement/(re) injury in chronic low back pain and its relation to behavioral performance. Pain 62:363-72

12. Woby SR, Roach NK, Urmston M, Watson PJ (2005). Psychometric properties of the TSK-11: a shortened version of the Tampa Scale for Kinesiophobia. Pain 117:137-44

13. Goubert L, Crombez G, Van Damme S, et al (2004) Confirmatory factor analysis of the Tampa Scale for Kinesiophobia. Invariant two factors models across low back pain patients and fibromyalgia patients. Clin J Pain 20:103-10

14. Roelofs J, Goubert L, Peters ML, et al (2004) The Tampa Scale for Kinesiophobia: further examination of psychometric properties in patients with chronic low back pain and fibromyalgia. Eur J Pain 8:495-502

15. Tkachuk GA, Harris CA (2012) Psychometric properties of the Tampa Scale for Kinesiophobia- 11 (TSK-11). J Pain 13:970-7

16. Schemer L, Schroeder A, Ornbol E, Glombiewski JA (2019) Exposure and cognitive- behavioural therapy for chronic back pain: An RCT on treatment processes. Eur J Pain 23:526-38

17. Malfliet A, Kregel J, Coppieters I, et al (2018) Effect of pain neuroscience education combined with cognition-targeted motor control training on chronic spinal pain a randomized clinical trial. JAMA Neurol 75:808-17

18. Paillé P, Mucchielli A (2016) L'analyse qualitative en sciences humaines et sociales. Armand Collin, Paris

19. Bunzli S, Smith A, Schutze R, O'Sullivan P (2015) The beliefs underlying pain-related fear and how they evolve: a qualitative investigation in people with chronic back pain and high painrelated fear. BMJ Open. 5:e008847. Doi: 10.1136/bmjopen-2015008847

20. Bunzli S, Smith A, Watkins R, et al (2015) What do people who score highly on the Tampa Scale of Kinesiophobia really believe? A mixed methods investigation in people with chronic nonspecific low back pain. Clin J Pain 31:621-32

21. Bunzli S, Smith A, Schutze R, et al (2017). Making sense of low back pain and pain-related fear. J Orthop Sports Phys Ther 47:628-36

22. Wideman TH, Asmudons GJG, Smeets RJEM, et al (2013) Rethinking the fear-avoidance model: toward a multidimensional framework of pain-related disability. Pain 154:2262-5

23. Rogers C (2018) Le développement de la personne. $2^{\mathrm{e}}$ éd. Interéditions, Paris 